\title{
Welche Rolle spielt die Gefährlichkeit für die Verlegung in eine sozialtherapeutische Einrichtung?
}

\section{Ein Plädoyer für die Modellierung vollzuglicher Selektionsprozesse}

\author{
Marcel Guéridon ${ }^{1}$ Stefan Suhling ${ }^{1}$ \\ Eingegangen: 15. Dezember 2019 / Angenommen: 20. Dezember 2019 / Online publiziert: 20. Februar 2020 \\ (c) Der/die Autor(en) 2020
}

\section{Zusammenfassung}

In Justizvollzugsanstalten müssen tagtäglich komplexe und mitunter folgenschwere Entscheidungen getroffen werden. Die Selektion in die sozialtherapeutische Behandlung ist ein typisches Beispiel für vollzugliche Prozesse, die auf solchen Entscheidungen beruhen. Einige jüngere Arbeiten haben die Qualität dieser Selektionsprozesse infrage gestellt, da keine Unterschiede hinsichtlich des A-priori-Rückfallrisikos erneuter schwerer Straftaten zwischen behandelten und unbehandelten Inhaftierten zu finden waren. Sowohl gemäß Risikoprinzip als auch gemäß der gesetzlichen Grundlage für die Selektion in die Sozialtherapie sollte jedoch ein höheres Risiko bei den Behandelten zu finden sein. Da diese Forschungsergebnisse allerdings lediglich auf einem Vergleich von behandelten und unbehandelten Personen beruhen, wirken einige Schlussfolgerungen voreilig, da die Selektion besser als Prozess verstanden und beschrieben werden sollte. Ein alternativer Ansatz wird vorgeschlagen, der auf Grundlage eines pragmatischen Modells ein besseres Verständnis der Selektionsprozesse verspricht. Eine Auswertung von Daten zur niedersächsischen Sozialtherapie weist entsprechend darauf hin, dass das Risiko erneuter Sexualstraftaten im Rahmen der Indikationsentscheidung eine prominente Rolle spielt und sich die Verweigerung der Behandlung nicht durch das Risiko vorhersagen lässt. Das Ausmaß der Unterschiede im mittleren Rückfallrisiko zwischen sozialtherapeutisch behandelten und nichtbehandelten Personen hängt zudem auch davon ab, ob Inhaftierte, die aufgrund bestimmter Merkmale konsistent keine Behandlung erhalten haben, in die Auswertung aufgenommen werden oder nicht.

Schlüsselwörter Selektionsprozesse $\cdot$ Deskriptive Forschung $\cdot$ Justizvollzug $\cdot$ Urteilsanalyse $\cdot$ Angezeigtheit

Marcel Guéridon, M.Sc.

Marcel.Gueridon@justiz.niedersachsen.de

1 Kriminologischer Dienst im Bildungsinstitut des niedersächsischen Justizvollzuges, Fuhsestraße 30, 29221 Celle, Deutschland 


\title{
What role does dangerousness play for the transfer to a social-therapeutic unit?
}

A plea for modelling selection processes in the prison system

\begin{abstract}
In prisons complex decisions, sometimes with serious consequences, have to be made on a daily basis. The selection into social-therapeutic treatment is a typical example for processes in the prison system that are based on such decisions. At the same time some recent publications have questioned the quality of the selection process as no differences could be found in the a priori risk of committing further offenses between treated and untreated inmates. Based on the risk principle as well as legal foundations for the selection into social-therapeutic treatment, a higher risk level should be found for the treated inmates. However, since these research results are merely based on a comparison of treated and untreated inmates, some conclusions seem to be rather premature as the selection should be understood and described as a process. An alternative approach based on a pragmatic model is presented, which promises a better understanding of the selection process. An evaluation of data on social therapy from Lower Saxony suggests that the risk of further sexual offenses plays a very prominent role in the decision on the indication for social-therapeutic treatment, whereas refusal of treatment could not be predicted by risk. Moreover, the differences in the average risk of recidivism between treated and untreated persons also depend heavily on the decision whether prisoners who are consistently excluded from treatment for particular reasons are included in the analysis.
\end{abstract}

Keywords Selection processes · Descriptive research · Prison system · Judgement analysis · Advisability

\section{Einleitung}

In Justizvollzugsanstalten müssen tagtäglich komplexe und mitunter folgenschwere Entscheidungen getroffen werden. Eine besondere Stellung nehmen Beurteilungen und Entscheidungen in der Form von Selektions- und Klassifikationsaufgaben ein, denn die vollzugliche Organisation ist an vielen Stellen im Grundsatz darauf ausgerichtet, Personen auf Grundlage bestimmter beobachtbarer Merkmale zu klassifizieren und zuzuweisen (Laubenthal 2015). Dabei ist nicht nur die passende Vollzugsform (offen/geschlossen) zu bestimmen, vielmehr muss während des gesamten Vollzugsverlaufs von der Behandlungsplanung über die Beurteilung von Selbst- oder Fremdgefährdung bis hin zur Gewährung von Lockerungen des Vollzugs immer wieder eine letztlich meist dichotome Entscheidung getroffen werden - häufig auf Grundlage unbestimmter Rechtsbegriffe auf der Tatbestandsseite und/oder explizit eingeräumtem Ermessenspielraum auf der Rechtsfolgenseite. Auch die Behandlungssteuerung gemäß dem Risk-Need-ResponsivityModell (RNR, Bonta und Andrews 2017), dem aktuell empirisch am besten abgesicherten Ansatz zur Behandlung straffälliger Personen, beruht wesentlich auf Klassifikation und Selektion. Besonders die Bestimmung der Gefährlichkeit, also der Wahrscheinlichkeit einer erneuten (schweren) Straftat, spielt für viele Entscheidungen im Vollzugsverlauf eine zentrale Rolle (Dahle 2005).

Ein wichtiges Beispiel ist die Selektion in die sozialtherapeutische Behandlung. Diese ist nicht nur eine typische Aufgabe des Justizvollzugs, sondern repräsentiert auch von ihrer Struktur her vollzugliche Selektionsprozesse in vielerlei Hinsicht. Sie wird wiederholt und reflektiert getroffen, weist nur begrenzte, letztlich dichotome Handlungsoptionen auf, muss in der Regel schriftlich begründet werden und ist mehrstufig strukturiert. Sie beruht ihrerseits auf einer Reihe von Urteils- und Entscheidungsprozessen, die auf verschiedene Personen verteilt sein können. Vor allem beruht die Entscheidung auch auf einer prognostischen Beurteilung des Risikos erneuter Straffälligkeit im Rahmen der Prüfung der „Angezeigtheit“. Vollzugliche Entscheidungen wie die Indikation einer sozialtherapeutischen Behandlung können damit wie viele Entscheidungen aus dem rechtswissenschaftlichen Kontext als „schwierig“ und kaum optimierbar aufgefasst werden, da a) die Konsequenzen oft unsicher sind, b) häufig Zielkonflikte vorliegen dürften und c) viele Informationen berücksichtigt werden müssen (Bröder und Hilbig 2017; Gigerenzer 2006).

Die normativen Grundlagen vollzuglicher Selektionsprozesse sind durchaus umfangreich aufgearbeitet und auch immer wieder Gegenstand gerichtlicher Überprüfung. Allerdings mangelt es selbst in der Frage der sozialtherapeutischen Behandlung, die sogar regelmäßig im Rahmen auch großer Evaluationsprojekte begleitet wird, an empirischen Ergebnissen zur tatsächlichen Selektionspraxis (Lösel et al. 2019, S. 10).

Dabei haben die wenigen empirischen Arbeiten zur Selektion in die sozialtherapeutische Behandlung durchaus irritierende Befunde erbracht. Sowohl Seifert (2014) als auch Endres (2014) berichten, dass in der Sozialtherapie bzw. der Behandlung für Sexualstraftäter allgemein nicht, wie erwartet, die Personen mit höherem Risiko erneuter Straffälligkeit und größeren „kriminogenen Bedarfen“ zu finden waren. Vielmehr waren die Risiko-Indizes (z. B. mittlere Static-99-Scores) der behandelten und der nicht behan- 
delten Inhaftierten vergleichbar. Dies würde dem Risikoprinzip im Sinne des RNR-Modells (Bonta und Andrews 2017) entgegenstehen, nach dem jene Personen besonders intensiv behandelt werden sollten, die ein erhöhtes Risiko erneuter Straffälligkeit aufweisen. Darüber hinaus würde eine entsprechende Selektionspraxis aber auch direkt infrage stellen, ob die Indikationskriterien Therapiebedürftigkeit und Therapienotwendigkeit korrekt berücksichtigt werden (Endres 2014).

Die naheliegende Interpretation einer mangelhaften Berücksichtigung des Risikoprinzips wäre jedoch voreilig. Im Rahmen des typischen Ansatzes, den auch Seifert (2014) und Endres (2014) nutzen, werden lediglich Personen, die eine Behandlung erhalten haben, mit Personen verglichen, bei denen dies nicht passiert ist. Mit diesem Ansatz wird jedoch der Prozesscharakter der Selektion übersehen, die im Verlauf eine ganze Reihe verschiedener Urteile und Entscheidungen umfasst. Wie schon Heckman und Smith (2004) betont haben, können verschiedene Faktoren an verschiedenen Stellen eines Prozesses ganz unterschiedliche Bedeutung haben. So könnte beispielsweise das erhöhte Risiko einer erneuten Straftat sehr wohl entscheidend für die Indikationsstellung sein, zugleich aber mit einer hohen Wahrscheinlichkeit einhergehen, dass die eigentlich für die Behandlung vorgesehene Person die Behandlung verweigert. In diesem Fall würde anhand des naiven Vergleichs korrekt geschlussfolgert werden, dass die Teilnehmer im Vergleich zu Nichtteilnehmern kein erhöhtes Risiko aufweisen. Dies wäre aber nun gerade kein fachliches oder entscheidungspolitisches Problem, sondern das Resultat einer nur begrenzt beeinflussbaren Selbstselektion. Wie dieses Beispiel zeigt, ist die Dekomposition des Selektionsprozesses eine Möglichkeit, scheinbar paradoxe oder zumindest kritisch zu bewertende Phänomene besser einordnen zu können. Eine Analyse auf Grundlage eines normativpragmatischen Modells der Selektion kann zudem davor schützen, ungeeignete Methoden zu verwenden, die letztlich fragliche Interpretationen begründen.

Von besonderer Bedeutung ist dabei die Unterscheidung von kompensatorischen und nonkompensatorischen Strategien der Urteilsbildung und Entscheidungsfindung. Kompensatorisch ist ein Modell bzw. eine Urteilsstrategie, wenn eine hohe Ausprägung eines Merkmals die Ausprägung eines anderen Merkmals auffangen kann. In einem nonkompensatorischen Modell führen dagegen bestimmte Merkmalsausprägungen immer zur Wahl einer bestimmten Option (Bröder 2000; Krohne und Hock 2015). Seifert (2014) unterstellt in ihrer Arbeit beispielsweise durch die Verwendung einer multiplen logistischen Regression zur Bestimmung der Determinanten der Behandlungsteilnahme zumindest implizit ein kompensatorisches Modell, in dem der Effekt einer zu geringen Strafdauer durch andere Merkmale aufgefangen werden kann. Tatsächlich war die empi- rische Wahrscheinlichkeit für die sozialtherapeutische Behandlung von Personen mit weniger als 24 Monaten Gesamtstrafe in ihren Analysen aber gleich null, was gegen die Angemessenheit eines kompensatorischen Modells spricht. ${ }^{1}$ Ihr statistisches Modell leidet entsprechend an einem völlig überhöhten Effekt für die Kategorien des Strafmaßes über 24 Monate. $^{2}$

Im Ergebnis ist ein erheblicher Mangel an deskriptiver Forschung zu vollzuglichen Selektionsprozessen zu konstatieren. Überdies wird in den wenigen überhaupt vorliegenden Arbeiten eine wenig ausgereifte Methodik verwendet und es fehlt durchaus an einem theoretischen Fundament. Im Folgenden wird daher auf Grundlage niedersächsischer Daten ein alternativer Ansatz präsentiert, um die Bedeutung der Gefährlichkeit im Prozess der Selektion in die Sozialtherapie detaillierter herauszuarbeiten. Um an die Arbeiten von Seifert (2014) und Endres (2014) anknüpfen zu können, beschränkt sich die Analyse auf die Indikation nach $\S 9$ Abs. 1 StVollzG. Deshalb werden nur Personen berücksichtigt, die wegen eines Sexualdelikts verurteilt wurden.

\section{Ein normativ-pragmatisches Modell der Selektion in die sozialtherapeutische Behandlung}

Die Prüfung der Indikation für eine sozialtherapeutische Behandlung erfolgte im hier betrachteten Zeitraum (2003-2007) in Niedersachsen im Rahmen der Behandlungsuntersuchung in einer zentralen Einweisungsabteilung. Dabei wurden im Rahmen der Indikationsprüfung gezielt Informationen anhand eines Indikationsbogens zusammengetragen. Die Indikation wurde im Ergebnis bejaht oder verneint, wobei explizit ausgewiesen wurde, wenn sich die verneinte Indikation auf aktuelle Bedingungen und Merkmale bezog, die sich im Vollzugsverlauf ändern können. Auf dieser Grundlage wurde im Rahmen der Vollzugsplanung die sozialtherapeutische Behandlung geplant. In Fällen mit langen Freiheitsstrafen wurde die Verlegung in der Regel auf einen späteren Zeitpunkt festgelegt. Ob die Verlegung schließlich umgesetzt wurde, hing zuletzt davon $a b$, ob die betroffene Person ihr zustimmte oder sie verweigerte.

\footnotetext{
${ }^{1}$ Es ist mitunter möglich, auch nonkompensatorische Modelle als lineare Modelle abzubilden (Cooksey 1996), aber dies ist im Fall von Seifert (2014) kein Thema.

2 Das Modell mag dennoch insofern korrekt geschätzt sein, als Seifert (2014) lediglich das Strafmaß der Bezugssache aufnimmt, nicht aber die Gesamtstrafe. Problematisch bleibt dies aber dennoch, da die Gesamtstrafe ja vorher empirisch als Ausschlusskriterium ermittelt wird, im zweiten Schritt aber weder konzeptuell noch empirisch im Modell berücksichtigt wird.
} 
Abb. 1 Modell der Selektion in die sozialtherapeutische Behandlung (Erläuterung siehe Text)

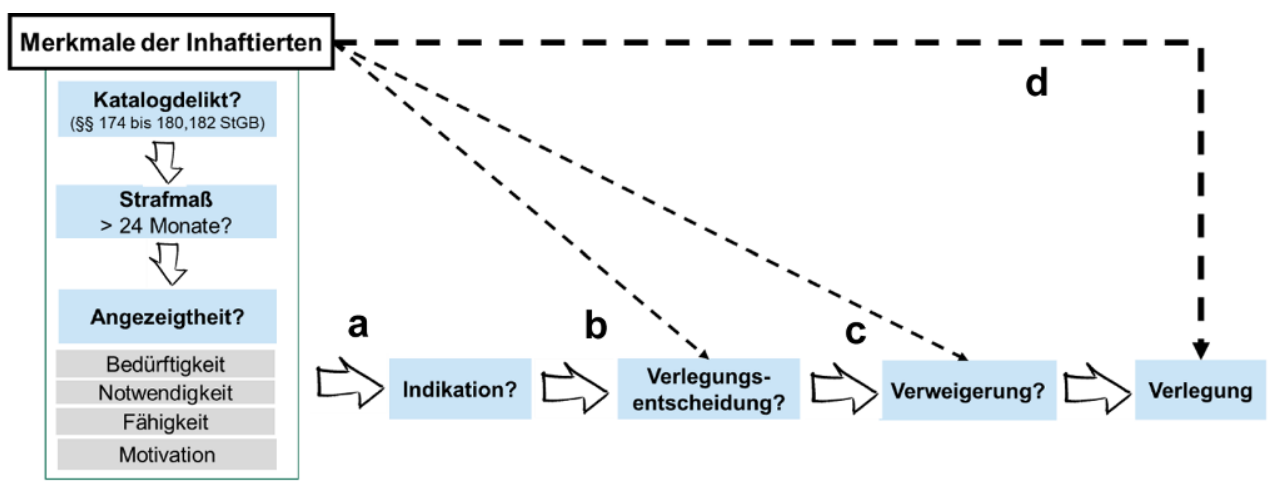

Im Ergebnis kann damit der in Abb. 1 dargestellte Selektionsprozess als Ausgangspunkt für die empirischen Analysen verwendet werden. Anhand dieses immer noch vereinfachenden Modells wird auch deutlich, wie der hier vorgestellte Ansatz die bisherigen Arbeiten erweitert. Statt nur die Merkmale der Inhaftierten mit der tatsächlichen Verlegung in Beziehung zu setzen (Pfad d), werden die entsprechenden Merkmale über den gesamten Prozess berücksichtigt. Daraus ergeben sich vier Beziehungen, die nur gemeinsam die Rolle der Gefährlichkeit im Selektionsprozess erschöpfend beschreiben können: a) Gefährlichkeit und Indikation, b) Gefährlichkeit und Verlegungsentscheidung/ Vollzugsplanung, c) Gefährlichkeit und Verweigerung sowie d) Gefährlichkeit und Verlegung/Behandlungsbeginn. Mithilfe der zur Verfügung stehenden Daten können im Folgenden die Fragestellungen a, $\mathrm{c}$ und d empirisch betrachtet werden.

\section{Fragestellung 1: Der Zusammenhang von Gefährlichkeit und Indikation}

Für die Prüfung der Angezeigtheit der Behandlung definiert $\S 9$ Abs. 1 StVollzG drei Bedingungen, die für die Indikation und Verlegung in eine sozialtherapeutische Einrichtung notwendig und in der Summe hinreichend sind. Bei Vorliegen der drei Kriterien liegt also kein Ermessenspielraum vor (Laubenthal 2015). Konkret muss eine Verurteilung wegen einer Katalogstraftat ( $\$ 174-180$ oder 182 StGB) vorliegen sowie eine zeitige Freiheitsstrafe von mehr als 24 Monaten verhängt worden $\operatorname{sein}^{3}$; zudem muss die Behandlung ,,angezeigt" sein. Das Delikt sowie die Strafzeit stellen formale

\footnotetext{
${ }^{3}$ Siehe Suhling und Wischka (2008) zur Frage des Bezugs auf Einzel- oder Gesamtstrafe. Seifert (2014) schreibt beispielsweise, dass in Sachsen-Anhalt auch bei Einzelstrafen unter 24 Monaten eine Indikation anzunehmen war, wenn die Gesamtstrafe über 24 Monaten liegt. Laubenthal (2015) vertritt genau die gegengesetzte Auffassung, dass in jenen Fällen § 9 Abs. 2 anzuwenden sei. Egg (2013) wiederum benennt das Strafmaß, ohne direkt auf die Frage von Einzel- oder Gesamtstrafe einzugehen.
}

Ausschlusskriterien dar, die entsprechend eine nichtkompensatorische Urteilsstrategie verlangen. Bei dem zentralen Begriff der „Angezeigtheit“ handelt es sich um einen unbestimmten Rechtsbegriff, der sich nicht direkt aus manifesten Merkmalen der Person ergibt, sondern ein Urteil höherer Ebene auf Grundlage mehr oder weniger bestimmbarer Konstrukte darstellt. In aller Regel wird die Angezeigtheit auf das Vorliegen der vier Konstrukte Therapiemotivation, Therapiefähigkeit, Therapiebedürftigkeit und Therapienotwendigkeit heruntergebrochen (Calliess und Müller-Dietz 2005; Egg 2013; Laubenthal 2015; Suhling und Wischka 2008). Die zentrale Frage der Modellierung ist im Fall des § 9 Abs. 1 StVollzG daher, wie genau das Urteil zur Angezeigtheit gebildet wurde und welche Faktoren dabei eine Rolle spielen. Eine kompensatorische Strategie würde bedeuten, dass die Therapiebedürftigkeit, deren Kern die Prognose des Risikos erneuter schwerer Straftaten bildet, immer einen Zusammenhang mit der Indikationsstellung aufweisen sollte. Es ist allerdings naheliegend, dass eine geringe Therapiefähigkeit und möglicherweise auch eine geringe Therapiemotivation im Rahmen einer nonkompensatorischen Urteilsstrategie unabhängig von der Therapiebedürftigkeit immer zu einer Ablehnung der Indikation geführt haben.

\section{Methode}

Für die Analyse der Indikationsentscheidung scheint entsprechend der dargestellten Grundlagen ein dreistufiges Vorgehen nötig. In einem ersten Schritt wird ein Modell der Therapiebedürftigkeit entwickelt. Dies bildet die Frage $a b$, ob das entsprechende Urteil erster Ordnung überhaupt relevante Merkmale abbildet. Im zweiten Schritt werden die zur Verfügung stehenden Merkmale dahingehend geprüft, ob bestimmte Ausprägungen möglicherweise nonkompensatorisch genutzt wurden, also immer zu einer Indikation oder iher Ablehnung geführt haben. Entsprechende Merkmalsausprägungen können über einfache Kreuztabellen ermittelt werden. Im letzten Schritt wird das Modell der Therapiebedürftigkeit auf die Indikationsentscheidung ge- 


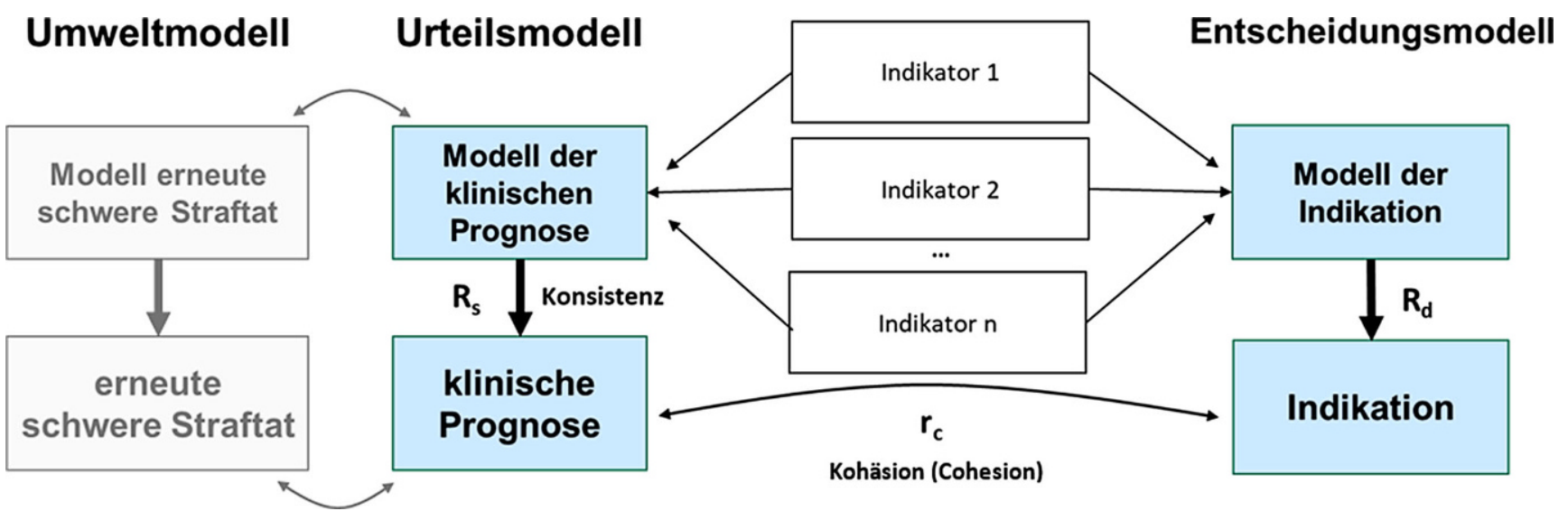

Abb. 2 Vereinfachte Darstellung eines bifokalen Linsenmodells. (Das Umweltmodell ist hier grau hinterlegt, da aufgrund von Anonymität keine Verknüpfung mit Daten zu erneuter Straffälligkeit möglich ist)

spiegelt, wobei die im zweiten Schritt ermittelten Personen ausgeschlossen werden, die aufgrund entsprechender Merkmale keine Chance hatten, eine Indikation zu erhalten. Für die Schritte 1 und 3 bietet sich eine Analyse in der Tradition des Linsenmodells nach Brunswik (1955) beziehungsweise der sozialen Urteilsbildungstheorie an (Cooksey 1996; Hammond 1996; Goldstein 2004). Dieser Ansatz zeichnet sich nicht nur durch hohe Flexibilität aus, sondern ermöglicht über die Dekomposition der Beziehungen anhand der „lens model equation“ (LME) auch die Bestimmung psychologisch interpretierbarer Parameter (Cooksey 1996; Stewart 2001; Hamm und Yang 2017). Konkret wird hier der Vorschlag von Beckstead (2017) aufgegriffen, im Rahmen eines bifokalen Linsenmodells Urteilsbildung und Entscheidung (Analyseschritte 1 und 3) gemeinsam in einem Modell abzubilden. Auf eine explizite Modellierung des Umweltmodells und damit der ökologischen Validität der Prognosen muss jedoch mangels Daten verzichtet werden. Das entsprechend vereinfachte bifokale Linsenmodell ist in Abb. 2 dargestellt.

\section{Erhebungsinstrument}

Für alle Inhaftierten mit Sexualdelikten wurde zwischen 2003 und 2007 in Niedersachsen eine Prüfung der Indikation anhand eines 6-seitigen Indikationsbogens vorgenommen. Da diese Informationen direkt im Kontext der Indikationsprüfung dokumentiert wurden, dürften sie in der Entscheidungssituation salient gewesen sein.

Der Indikationsbogen war in sechs Abschnitte unterteilt. Im ersten Abschnitt wurden einigen Rahmendaten (Name, Datum der Prüfung u. Ä.) abgefragt, die hier nicht weiter von Belang sind. Der zweite Abschnitt umfasst eine Aktenauswertung zu kriminologischen Merkmalen (Strafbeginn, Strafende, Strafhöhe, Anordnung einer Maßregel, Schuldfähigkeit) sowie eine Einordnung des Indexdelikts und schließlich zwei Indexwerte zu Allgemein- und Sexualkriminalität, bei denen aus jeweils fünf Merkmalen ein Summenwert zwischen 1 und 7 gebildet wurde, wobei ein hoher Wert einer höheren Belastung entspricht. Im Weiteren wurden testpsychologische Daten erfasst, welche jedoch häufig fehlen. In einem weiteren Abschnitt wurden die Ergebnisse der drei Prognoseverfahren SVR-20, PCL:SV und RRS sowie das Vorliegen von psychiatrischen Störungen (Achse-I des DSM-IV) oder Persönlichkeitsstörungen dokumentiert. ${ }^{4}$ Im letzten Abschnitt wurde eine Reihe klinischer Urteile zur Wahrscheinlichkeit erneuter Straffälligkeit (neues Sexualdelikt, erneute Inhaftierung, erneute Straftat ohne Sexualdelikt), der Fluchtgefahr, der Tatleugnung, der Behandlungsmotivation und der Behandlungsmöglichkeit abgefragt. Diese Urteile wurden 3- bis 4-stufig erfasst, wobei die Kategorien grundsätzlich als ordinal skaliert aufgefasst werden können. Die klinischen Einschätzungen zum Risiko können für die vorliegende Fragestellung sinnvoll als „Proxy“-Variable für die Therapiebedürftigkeit interpretiert werden. Schließlich sollten ggf. Gegenindikationen benannt werden, die gegen eine sozialtherapeutische Behandlung sprachen, bevor eine Entscheidung über die Indikation getroffen wurde. Diese wurde in drei Kategorien erfasst: Indikation gegeben (auch wenn Strafrest noch zu lang), Indikation nicht gegeben sowie Indikation aktuell nicht gegeben.

\section{Stichprobe}

Zur Verfügung stehen Daten von insgesamt $n=456$ Indikationsprüfungen. Es handelt sich durchweg um erwachse-

\footnotetext{
${ }^{4}$ Die in diesem Beitrag erwähnten Prognoseverfahren (SVR-20, RRS, PCL:SV, Static-99, OGRS-3) werden als bekannt vorausgesetzt und nicht weiter erläutert. Für alle Verfahren sei auf die jeweilige Darstellung in Rettenberger und von Franqué (2013) verwiesen.
} 
ne Männer, die zum Zeitpunkt der Indikationsprüfung zwischen 24 und 76 Jahre alt waren. Die Mehrheit war wegen eines sexuellen Missbrauchs von Kindern verurteilt (52,4\%, davon in 3 von 5 Fällen mit innerfamiliärem Opfer), weitere 41,0\% wegen eines Delikts gegen Erwachsene und 6,6\% wegen anderer oder nicht eindeutig bestimmbarer Delikte. Die geringste verhängte Strafdauer lag bei 1,5 Jahren; im Mittel wurden 4,5 Jahre (Median: 4 Jahre) verhängt. Lebenslange Freiheitstrafen $(n=3)$ wurde dabei mit einem Strafmaß von 15 Jahren einbezogen.

Im Ergebnis wurde in 286 Fällen die Indikation bejaht $(62,7 \%)$ und in 134 Fällen verneint $(29,4 \%)$. In weiteren 36 Fällen $(7,9 \%)$ wurde die Indikation als ,derzeit nicht gegeben“ bewertet. Für die folgenden Auswertungen werden die letzteren beiden Kategorien zur Vereinfachung zusammengefasst.

\section{Ergebnisse}

Modell der Therapiebedürftigkeit Als Proxy für die Einschätzung der Therapiebedürftigkeit wird die klinische Vorhersage des Risikos einer erneuten Sexualstraftat verwendet. Diese Einschätzung zeigt die höchste Korrelation mit der Indikationsentscheidung $(\rho=0,29, p<0,001$, Gesamtstichprobe). Die anderen beiden klinischen Urteile zum Risiko erneuter Straftaten zeigen dagegen keinen Zusammenhang mit der Indikationsentscheidung (Prognose erneute Inhaftierung: $\rho=0,07, p=0,12$, Prognose anderes Delikt: $\rho=0,01, p=0,90)$. Obwohl das Urteil ordinal erfasst wurde (gering, gering bis offen, offen, hoch), scheint hier eine lineare Modellierung vertretbar. ${ }^{5}$

Der beste Prädiktor für das klinische Urteil zur Wahrscheinlichkeit einer neuen Sexualstraftat wiederum ist die (klinisch bestimmte) Risikokategorie des SVR-20 (bivariat: Cramers V $=0,52, p<0,001)$. Dennoch wird im Detail auch deutlich, dass die Diskrepanz zwischen den beiden (klinischen) Einschätzungen nicht nur auf die unterschiedliche Zahl an Kategorien zurückzuführen ist, sondern weitere Informationen berücksichtigt worden sein müssen. Wenig überraschend korrelieren die möglichen Prädiktoren untereinander recht hoch (z.B. RRS-K-Wert und SVR20-Summenwert zu $\mathrm{r}=0,50, p<0,001)$.

Aus Platzgründen wird hier lediglich das finale Regressionsmodell dargestellt. Es basiert auf einer Vorauswahl plausibler Indikatoren, die weniger als $10 \%$ fehlende Werte aufweisen. Diese Vorauswahl wurde durch eine rückwärts gerichtete schrittweise Regressionsanalyse weiter auf die besten Prädiktoren reduziert. Es fanden sich keine Hinweise auf einen Zusammenhang fehlender Werte mit der Indikationsstellung, weshalb Personen mit fehlenden Wer-

\footnotetext{
5 Ein ergänzend berechnetes ordinales Regressionsmodell zeigt keine abweichenden Ergebnisse.
}

ten ausgeschlossen wurden. Darüber hinaus können in diesem Auswertungsschritt aber noch alle Fälle eingehen. Da ein möglichst prädiktives, aber noch einigermaßen robustes Modell gesucht wird, schien diese Vorgehensweise gerechtfertigt. Dennoch sollten nicht unbedingt die einzelnen Koeffizienten im Detail interpretiert werden, sondern vielmehr das Gesamtbild.

Wie Tab. 1 (rechte Seite, Urteilermodell) zeigt, lässt sich die klinische Prognose erneuter Sexualdelinquenz gut durch die Ergebnisse der Prognoseverfahren (SVR-20, RRS-K) vorhersagen. Darüber hinaus zeigen auch die aus verschiedenen kriminologischen Merkmalen gebildeten Indexwerte konsistente Zusammenhänge, wobei hohe Punktwerte im Index für Sexualkriminalität mit einer schlechteren Prognose einhergehen, ein höherer Punktwert im Index für Allgemeinkriminalität jedoch mit einer günstigeren Prognose des Risikos erneuter Sexualdelinquenz. Darüber hinaus wurde bei höherem Alter eher eine schlechtere Prognose gestellt, bei Vorliegen einer psychiatrischen Störung dagegen eine bessere.

Ausschlusskriterien Zur Bestimmung nonkompensatorisch genutzter Informationen, die immer zu einer Ablehnung der Indikation geführt haben, wurden die zur Verfügung stehenden Merkmale anhand von Kreuztabellen deskriptiv ausgewertet. Im Ergebnis zeigt sich, dass die meisten Merkmale scheinbar kompensatorisch genutzt wurden, wenn sie denn im Rahmen der Urteilsbildung genutzt wurden. Dies trifft etwa auf das Vorliegen einer psychiatrischen oder Persönlichkeitsstörung zu, die nicht in jedem Fall zu einer Ablehnung der Indikation geführt haben. Auch ein hohes Maß an Tatleugnung bzw. eine Einschätzung als „nicht motivierbar“ führte nicht zwangsläufig zu einer Ablehnung der Indikation. Gleiches gilt für Personen mit einem Strafrest unter 24 Monaten, jedoch nicht für die Personen mit einem Gesamtstrafmaß von unter 1,5 Jahren Freiheitsstrafe. In keinen Fall wurde eine Indikation für eine sozialtherapeutische Behandlung gestellt, wenn eine Maßregel nach $\S \S 63$ oder 64 StGB verhängt worden war. Auch im Fall einer drohenden oder anstehenden Abschiebung sowie einer Drogenabhängigkeit wurde die Indikation durchgehend verneint, im Fall einer Alkoholabhängigkeit nur in einem Einzelfall bejaht. Konsistent wurde auch im Fall fehlender Behandlungsnotwendigkeit (,andere Maßnahmen ausreichend“) keine Indikation gestellt. Ebenfalls in keinem Fall haben Personen mit einem Intelligenzquotienten unter 67 oder fehlenden deutschen Sprachkenntnissen eine Indikation erhalten. Neben diesen detailliert gemessenen Merkmalen wurde auch ein allgemeines Urteil zur „Behandlungsmöglichkeit“" abgefragt. Während die Kategorie „Behandlung kaum/ eingeschränkt möglich“ in $55 \%$ der Fälle zu einer Indikation geführt hat, wurde die Indikation nur in 2 von 40 Fällen bei Auswahl der Kategorie „Behandlung nicht möglich“ ge- 
Tab. 1 Tabellarische Darstellung des Linsenmodells

\begin{tabular}{|c|c|c|c|c|c|c|}
\hline \multicolumn{6}{|c|}{ Kohäsion: $\rho=37$} & \\
\hline \multirow{13}{*}{$\begin{array}{l}\text { Indika- } \\
\text { tion }\end{array}$} & \multicolumn{3}{|c|}{ Entscheidungsmodell } & & \multirow{2}{*}{$\begin{array}{l}\text { Urteilsmodell } \\
(n=379)\end{array}$} & \multirow{13}{*}{$\begin{array}{l}\text { Klin. } \\
\text { Prognose } \\
\text { erneutes } \\
\text { Sexualdelikt }\end{array}$} \\
\hline & $\begin{array}{l}\text { Modell } 3 \\
(n=308)\end{array}$ & $\begin{array}{l}\text { Modell } 2 \\
(n=309)\end{array}$ & $\begin{array}{l}\text { Modell } 1 \\
\left(\mathrm{n}_{\min }=308\right.\end{array}$ & & & \\
\hline & OR & & & & $\beta$ & \\
\hline & $3,63 * * *$ & - & $3,16^{* * *}$ & Klin. Prognose erneutes Sexualdelikt & - & \\
\hline & 1,57 & $2,28 * *$ & $2,77 * * *$ & Klin. Risikobeurteilung SVR-20 & $0,27 * *$ & \\
\hline & 1,00 & 1,02 & $1,02^{(*)}$ & Alter & $0,10 * *$ & \\
\hline & 0,98 & 0,97 & 1,00 & $\begin{array}{l}\text { Summenwert Index Allgemeinkriminali- } \\
\text { tät }\end{array}$ & $-0,15 * * *$ & \\
\hline & 1,01 & 1,06 & $1,10 * *$ & Summenwert Index Sexualkriminalität & $0,20 * * *$ & \\
\hline & 0,99 & 1,03 & $1,09 * * *$ & Summenwert SVR-20 & $0,24 * * *$ & \\
\hline & 0,92 & 1,03 & $1,16^{* * *}$ & RRS-K-Wert & $0,33^{(*)}$ & \\
\hline & 0,64 & $0,52^{(*)}$ & 0,94 & Psychiatr. Störung? & $-0,07 * * *$ & \\
\hline & \multicolumn{3}{|c|}{$\mathrm{R}^{2}$ (Nagelkerke) } & & Korr. $\mathrm{R}^{2}=0,54$ & \\
\hline & 0,28 & 0,17 & - & & & \\
\hline
\end{tabular}

Rechte Seite (klin. Prognose erneutes Sexualdelikt): lineare Regression der Prognose auf die Indikatoren (Urteilsmodell), Gesamtstichprobe Linke Seite (Indikation): logistische Regression der Indikationsentscheidung auf die Indikatoren (Entscheidungsmodell) unter Ausschluss von Personen mit formalen oder empirisch ermittelten Ausschlusskriterien. Modell 1: bivariate Zusammenhänge, Modell 2: multiples log. Regressionsmodell, exkl. klin. Prognose erneuter Sexualstraftat, Modell 3: multiples log. Regressionsmodell, inklusive. klin. Prognose erneuter Sexualstraftat OR Odds-Ratio

Ohne $* p \geq 0,10,{ }^{*} p p<0,10, * p<0,05, * * p<0,01, * * * p<0,001$

stellt. Selbst hier scheint also zumindest in Ausnahmefällen ein kompensatorisches Urteil gefällt worden zu sein.

Therapiebedürftigkeit und Indikation Wie schon erwähnt, beträgt der Zusammenhang der klinischen Prognose einer erneuten Sexualstraftat und der Indikation in der Gesamtstichprobe $\rho=0,29 \quad(p<0,001$, Odds-Ratio 2,1). Werden Personen ausgeschlossen, bei denen eine Abschiebung, Alkohol- oder Drogenkonsum, ein Strafmaß unter 1,5 Jahren, eine Maßregel nach den $\S \S 64$ oder 65 StGB, ein IQ unter 67, fehlende Sprachkenntnisse oder eine fehlende Behandlungsmöglichkeit dokumentiert wurde (Schritt 2), erhöht sich die Korrelation auf $\rho=0,37(p<0,001$, OddsRatio 3,2).

Auf die Indikation bezogen zeigen lediglich die Indikatoren für die Sexualdelinquenz relevante Zusammenhänge (SVR-20-Risikokategorie, SVR-20-Punktwert, RRS-KWert, Indexwert Sexualdelinquenz), nicht aber der Indexwert für Allgemeindelinquenz (Tab. 1, linke Seite, Modell 1). In Rahmen eines multiplen logistischen Regressionsmodell dominierte die klinisch bestimmte Risikokategorie im SVR-20 als bester Prädiktor, während die übrigen Merkmale darüber hinaus kaum einen inkrementellen Beitrag leisten (Tab. 1, linke Seite, Modell 2). Auch die SVR-20-Risikokategorie verliert ihren prädiktiven Wert, wenn die klinische Prognose der erneuten Straffälligkeit berücksichtigt wird (Tab. 1, linke Seite, Modell 3).

\section{Zwischenfazit}

Die dargestellten Auswertungen weisen darauf hin, dass die klinische Prognose eines erneuten Sexualdelikts als scheinbar zentrale Dimension der Therapiebedürftigkeit ein wichtiger Prädiktor der Indikationsentscheidung ist. Dieser Effekt ist stärker ausgeprägt, wenn Personen ausgeschlossen werden, die aufgrund geringer Behandlungsfähigkeit oder anderer empirisch ermittelter Ausschlusskriterien in keinem Fall eine Indikation erhalten haben.

Das klinische Urteil eines erneuten Sexualdelikts lässt sich gut durch Indikatoren vorhersagen, deren ökologische Validität angenommen werden kann, v. a. durch die Ergebnisse validierter Prognoseverfahren. Das klinische Urteil kann daher im Rahmen der vorliegenden Auswertungen grundsätzlich als sinnvolles Maß für die Behandlungsbedürftigkeit herangezogen werden. Dennoch bleibt ein wesentlicher Teil der Varianz unaufgeklärt; es werden also über die verfügbaren Indikatoren hinaus weitere Informationen für die Prognose herangezogen.

Insgesamt kann festgehalten werden, dass im Rahmen der Indikationsstellung die Behandlungsbedürftigkeit in Form der Erwartung erneuter Sexualdelikte umfassend einbezogen wird. Insofern scheint die Angezeigtheitsprüfung das Risikoprinzip zu berücksichtigen, wenngleich dieses anscheinend von den Beurteilern und Beurteilerinnen lediglich auf erneute Sexualdelinquenz bezogen wurde. 


\section{Fragestellung 2: Der Zusammenhang von Gefährlichkeit und Verweigerung}

Im Rahmen des dargestellten Selektionsprozesses in die sozialtherapeutische Behandlung stellt die mögliche Verweigerung der Verlegung durch die Inhaftierten einen wichtigen Punkt dar. Während es in der Frage der Berücksichtigung der Therapiebedürftigkeit im Rahmen der Indikationsstellung wesentlich um die Qualität und Konsistenz vollzuglicher Urteile und Entscheidungen geht, stellt die Verweigerung eine nur begrenzt beeinflussbare Selbstselektion dar. Es kann vermutet werden, dass Personen mit einem hohen Risiko erneuter schwerer Straftaten zugleich eine erhöhte Neigung aufweisen, die Verlegung zu verweigern. Diese These lässt sich relativ einfach überprüfen, indem die Verweigerer auf risikorelevanten Merkmalsdimensionen mit den Personen verglichen werden, die die Verlegung nicht verweigert haben.

\section{Datengrundlage und Stichprobe}

Für diesen Schritt der Auswertung kann auf einen zweiten Datensatz zurückgegriffen werden, der sich aufgrund der kompletten Anonymität leider nicht mit den Daten zur Indikation verknüpfen lässt. Es handelt sich hierbei um einen Datensatz zur Evaluation der Sozialtherapie in Niedersachsen. Dieser beruht für die sozialtherapeutisch behandelten Personen v.a. auf einer Basisdokumentation, die um Daten aus Aktenanalysen ergänzt wurden. Im Fall der Personen, die nicht sozialtherapeutisch behandelt wurden, wurden die Daten komplett aus den Gefangenenpersonalakten erhoben. Es handelt sich hierbei mit hoher Wahrscheinlichkeit fast um eine Totalerhebung aller Personen, die im Zeitraum zwischen 2003 und 2008 aus dem niedersächsischen Justizvollzug entlassen wurden und auch wegen eines Sexualdelikts verurteilt worden waren. Es dürfte daher eine wesentliche Überschneidung mit der Stichprobe der Indikationsdaten geben. Der Datensatz ist sehr umfangreich und wird daher hier nicht im Detail beschrieben; wichtig für die vorliegende Fragestellung sind lediglich die erhobenen risikorelevanten Merkmale. Neben dem Ergebnis des Static99 liegt das anhand des OGRS-3 geschätzte Risiko einer erneuten Straftat innerhalb von 24 Monaten vor.

Die Stichprobe besteht aus insgesamt 369 männlichen Personen. In 72 Fällen (19,5\%) handelte es sich um Personen, deren Freiheitsstrafe im Jugend- oder Jungtätervollzug vollstreckt wurde. Insgesamt 252 Personen $(68,3 \%)$ wurden zumindest zeitweise sozialtherapeutisch behandelt. Für insgesamt 31 Personen wurde dokumentiert, dass die sozialtherapeutische Behandlung aufgrund einer Verweigerung nicht zustande kam, was 26,5\% aller nichtbehandelten Personen ausmacht.

\section{Ergebnisse}

Der mittlere Static-99-Wert für die sozialtherapeutisch behandelten Personen lag bei 2,7, das anhand des OGRS-3 ermittelte allgemeine Risiko erneuter Straftaten innerhalb von 24 Monaten lag im Mittel bei 35,7\%. Demgegenüber lag der Static-99-Wert bei den Verweigerern im Mittel bei 2,3 und das anhand des OGRS-3 ermittelte Risiko bei 32,0\%.

Eine einfaktorielle ANOVA ergab für den Gruppenvergleich weder für die Static-99-Werte $(\mathrm{F}(1,282)=0,981$, $\left.p=0,32, \quad \eta^{2}=0,003\right)$ noch für die OGRS-3-Werte $\left(F(1,277)=0,892, p=0,35, \eta^{2}=0,004\right)$ ein signifikantes Ergebnis.

\section{Zwischenfazit}

Eine mögliche Erklärung für die in der Literatur gefundenen fehlenden Unterschiede hinsichtlich der Therapiebedürftigkeit zwischen sozialtherapeutisch behandelten und nicht sozialtherapeutisch behandelten Personen, die wegen einer Sexualstraftat verurteilt wurden (Endres 2014; Seifert 2014), besteht in einem systematischen Selektionseffekt durch die Verweigerung.

In den vorliegenden Daten konnte ein entsprechender Zusammenhang allerdings nicht bestätigt werden. Weder hinsichtlich des Risikos eines erneuten Sexualdelikts noch hinsichtlich des allgemeinen Risikos erneuter Straftaten konnten Auffälligkeiten bei Personen gefunden werden, die die Behandlung verweigert haben. Auch absolut betrachtet handelt es sich nicht um Personen mit auffällig hohen Werten in den verwendeten Prognoseverfahren. Ein gewisser Selektionseffekt lässt sich damit dennoch nicht ausschließen, da auch ein vergleichbares Risikoniveau den Mittelwert in die Richtung des Niveaus der Behandelten verzerren kann. Schon theoretisch ist eine Zuordnung der Verweigerer zur Vergleichsgruppe nur unter bestimmten Umständen angemessen, schließlich hätten diese Personen nach Plan eigentlich behandelt werden sollen. Für die hier thematisierten Fragestellungen scheint es tatsächlich sinnvoller, die Verweigerer auszuschließen oder gar zur Gruppe der „Behandelten“ zu zählen.

\section{Fragestellung 3: Der Zusammenhang von Gefährlichkeit und Verlegung}

\section{Fragestellung und Methode}

Im letzten Schritt bleibt der Vergleich der tatsächlich verlegten Personen mit den nichtverlegten Personen, was dem Ansatz von Endres (2014) und Seifert (2014) entspricht. Für diese Fragestellung kann erneut auf den bereits unter Fragestellung 2 beschriebenen Datensatz zur Evaluation 
Tab. 2 Vergleich risikorelevanter Merkmale zwischen sozialtherapeutisch Behandelten und nichtverlegten Personen

\begin{tabular}{llllll}
\hline & $n$ & $\begin{array}{l}\text { Static-99 } \\
\text { M (SD) }\end{array}$ & $\begin{array}{l}\text { OGRS-3 } \\
\text { M (SD) }\end{array}$ & $\begin{array}{l}\text { Static-99 } \\
\text { (Welch-Test) }\end{array}$ & $\begin{array}{l}\text { OGRS-3 } \\
\text { (F-Test) }\end{array}$ \\
\hline Behandelte & 252 & 2,7 & 35,6 & - & - \\
& & $(2,1)$ & $(20,3)$ & & \\
Nichtverlegte & 117 & 2,4 & 40,2 & $2,3(1,283,5)$, & $3,7(1,367)$, \\
& & $(1,6)$ & $(22,2)$ & $p=0,129$ & $p=0,055$ \\
Nichtverlegte & 36 & 2,2 & 41,4 & $2,5(1,53,2)$, & $2,5(1,285)$, \\
(ohne Personen mit Ausschlussgründen) & & $(1,6)$ & $(21,5)$ & $p=0,118$ & $p=0,118$ \\
$\begin{array}{l}\text { Nichtverlegte } \\
\text { (nur Personen mit fehlender Notwendigkeit) }\end{array}$ & 31 & 2,1 & 42,9 & $4,5(1,44,9)$, & $3,8(1,280)$, \\
\end{tabular}

Die Teststatistiken beziehen sich jeweils auf einen Vergleich zur Gruppe der sozialtherapeutisch behandelten Personen. Da im Fall der Static-99Werte gemäß signifikanter Levene-Tests keine Varianzhomogenität angenommen werden, wurde die Welch-Statistik herangezogen. Im Fall der OGRS-3-Werte konnte Varianzhomogenität angenommen werden, weshalb die F-Statistik genutzt wurde

$M$ Mittelwert, $S D$ Standardabweichung

der Sozialtherapie zurückgegriffen werden. Dabei soll zuerst ohne Einschränkung die gesamte Gruppe der Nichtbehandelten herangezogen werden. In einem zweiten Schritt werden dann jene Personen aus der Analyse herausgenommen, die eine Verlegung verweigert haben oder einen empirischen Ausschlussgrund aufweisen. Dies betrifft Personen mit zu kurzem Strafrest, einer Substanzmittelproblematik, einer drohenden Abschiebung sowie zu geringen sprachlichen oder intellektuellen Fähigkeiten.

\section{Ergebnisse}

Der einfache Vergleich zeigt keine signifikanten Unterschiede hinsichtlich des mittleren Static-99-Werts (WelchStatistik $=2,3(1,283,5), p=0,129)$. Die nicht sozialtherapeutisch behandelten Personen weisen ein marginal signifikantes höheres Risiko erneuter Straftaten auf (gemessen am OGRS-3-Wert; $\mathrm{F}=3,7(1,367), p=0,055)$.

Werden lediglich die Personen berücksichtigt, bei denen kein formaler oder empirischer Ausschlussgrund vorliegt, reduziert sich die Vergleichsgruppe auf 36 Personen, von denen 31 explizit wegen fehlender Behandlungsnotwendigkeit (,andere Maßnahmen ausreichend“) nicht indiziert wurden. Wie Tab. 2 im Detail zeigt, weist dieser Personenkreis zwar ein vergleichbares bis sogar erhöhtes Risiko allgemeiner Kriminalität auf (OGRS-3), aber ein tendenziell geringeres Risiko erneuter Sexualdelinquenz (Static-99). Werden nur die Personen herangezogen, die wegen mangelnder Behandlungsnotwendigkeit keine Indikation erhalten haben, zeigt sich der eigentlich erwartete Unterschied, der für ein höheres Risiko erneuter Sexualdelinquenz unter den sozialtherapeutisch behandelten Personen spricht (Welch-Statistik $=4,5(1,44,9), p=0,040)$.

\section{Zwischenfazit}

Die Auswertung der Daten anhand des auch von Endres (2014) und Seifert (2014) verfolgten einfachen Ansatzes,
Behandelte und Nichtbehandelte hinsichtlich risikorelevanter Merkmale zu vergleichen, führt auf den ersten Blick zu vergleichbaren Ergebnissen. Auch in der niedersächsischen Stichprobe findet sich im Rahmen dieser Methodik kein Unterschied hinsichtlich des Risikos erneuter Sexualstraftaten, jedoch sogar ein tendenziell höheres Risiko für allgemeine Kriminalität aufseiten der nicht sozialtherapeutisch behandelten Personen.

Werden allerdings formale und empirisch ermittelte Ausschlusskriterien berücksichtigt, zeigen sich gewisse Differenzen. Wer explizit wegen mangelnder Behandlungsnotwendigkeit nicht verlegt wurde, weist zumindest tendenziell auch ein geringeres Risiko erneuter Sexualdelinquenz auf.

Ein generell bemerkenswerter Befund ist dabei, dass nur etwa ein Drittel der nicht sozialtherapeutisch behandelten Personen kein formales oder empirisch ermitteltes Ausschlusskriterium aufweist. Dieses Muster spricht für eine insgesamt sehr hohe Selektionsrate. Scheinbar wurde wirklich nur in wenigen Fällen die Indikation auf Basis einer geringen Therapiebedürftigkeit verneint. Dafür spricht, dass auch bei niedrigen Static-99-Werten überwiegend eine sozialtherapeutische Behandlung zumindest begonnen wurde. Diese Situation mag auch darauf zurückzuführen sein, dass in Niedersachsen ausreichende Behandlungskapazitäten vorgelegen zu haben scheinen. Zumindest ließ sich auf Aktenbasis nicht ermitteln, dass Personen wegen Mangel an Kapazität keine Behandlung erhalten haben.

\section{Diskussion}

Ausgehend von den durchaus überraschenden Ergebnissen bisheriger Vergleiche des Risikos erneuter schwerer Straftaten zwischen sozialtherapeutisch behandelten und nichtbehandelten Personen wurde dafür plädiert, auf Grundlage eines Modells des Selektionsprozesses über den direkten 
Vergleich hinaus die Indikationsentscheidung und die Behandlungsverweigerung explizit einzubeziehen.

Im Ergebnis zeigt sich für Niedersachsen, dass im Rahmen der Indikationsstellung das Risiko einer erneuten Sexualstraftat eine sehr prominente Rolle einnahm. Die Beurteiler und Beurteilerinnen scheinen bei der Frage der Therapiebedürftigkeit dabei das einschlägige Delikt vor Augen zu haben, nicht etwa eine erneute Inhaftierung oder allgemeine Delinquenz. Dieser Zusammenhang wird noch deutlicher, wenn Personen aus den Analysen ausgeschlossen werden, die bestimmte Merkmale oder Merkmalskombinationen aufweisen, die empirisch immer zu einer Ablehnung der Indikation geführt haben. Dieser Befund stützt die Forderung, normativ und theoretisch fundierte Auswertungen durchzuführen, die nonkompensatorische Urteils- und Entscheidungsstrategien in den Selektionsprozessen berücksichtigen.

Keine Bestätigung fand die These, dass ein erhöhtes Risiko mit einer Neigung zur Verweigerung der Behandlung einhergeht. Eine Verzerrung durch die Selbstselektion der Inhaftierten ist daher zumindest für die Daten aus Niedersachsen nicht in größerem Maß zu erwarten. Dennoch sollten Verweigerer grundsätzlich im Rahmen von Vergleichen zwischen behandelten und unbehandelten Personen gesondert betrachtet, herausgenommen oder u. U. sogar der Gruppe der Behandelten zugeordnet werden.

Auch die Auswertung der niedersächsischen Daten zeigte indes bei einem einfachen Vergleich der sozialtherapeutisch behandelten und unbehandelten Inhaftierten keinen signifikanten Unterschied zwischen den Verlegten und den Nichtverlegten hinsichtlich der verfügbaren Risikoindikatoren. Werden jedoch Personen mit formalen oder zumindest konsistent genutzten Ausschlusskriterien von der Analyse ausgeschlossen, zeigt sich ein höheres mittleres Risiko erneuter Sexualstraftaten unter den Behandelten. Eben dies war bei Berücksichtigung des ermittelten Indikationsmodells auch zu erwarten.

Obwohl hier nur die Rolle des Risikos erneuter Straffälligkeit berücksichtigt wurde, scheint uns der hier verfolgte Ansatz ein großes Potenzial zu bieten, mehr über die vollzuglichen Selektionsprozesse zu lernen als durch reine Vergleiche von Behandelten und Unbehandelten. Besonders betont werden sollte dabei, dass ein großer Teil der nicht behandelten Personen wegen formaler oder anderer konsistent genutzter Ausschlusskriterien keine Indikation erhalten hat. $\mathrm{Ob}$ diese Personen im Rahmen der Evaluationsforschung als Kontroll- oder Vergleichsprobanden herangezogen werden können, sollte zumindest kritisch hinterfragt werden.

Natürlich ist der hier vorgestellte Ansatz kein Allheilmittel, und auch die hier präsentierten Auswertungen weisen Schwächen auf. Obwohl sich das Linsenmodell und die soziale Urteilsbildungstheorie auch hier als sinnvolles Rahmenmodell für die Auswertungen bewährt haben, konnte das Potenzial nicht ansatzweise ausgeschöpft werden. Statt der hier präsentierten Auswertungen über verschiedene Beurteiler und Beurteilerinnen hinweg wäre eigentlich eine statistisch-idiografische Analyse Mittel der Wahl, indem die Urteils- und Entscheidungsmodelle auf Ebene der jeweiligen Person erstellt und verglichen werden. Es ist nicht davon auszugehen, dass alle an den hier berücksichtigten Indikationsentscheidungen beteiligten Personen eine identische Urteilsstrategie nutzten. Insofern scheint ein wichtiger Schritt für die Zukunft zu sein, bei der Datenerhebung die beurteilende Person (anonym) mitzuerfassen.

Bedauerlich ist zudem, dass die Indikationsdaten nicht mit den Daten aus der Evaluation verknüpft werden konnten. Eine durchgehende Modellierung von der Indikation bis zu Verlegung und darüber hinaus bis zum Abbruch oder zum Abschluss der Maßnahme bietet größeres Potenzial als die hier dargestellte Auswertung auf Grundlage verschiedener Datensätze. Eine weitere Einschränkung ist, dass die Vollzugsplanung ( $\mathrm{fad} b$, Abb. 1) nicht berücksichtigt werden konnte. Gerade in diesem Rahmen ist es möglich, dass eine bestimmte Entscheidungspolitik verzerrende Effekte erzeugt.

Methodisch geht mit den durch Aktenanalyse gewonnenen Daten das grundsätzliche Problem einher, dass nur offizielle Angaben und Begründungen verwendet werden konnten. In Einzelfällen wie auch systematisch ließen sich ohne größere Schwierigkeit apokryphe Gründe gegen oder für eine Indikation in solchen Verwaltungsdokumenten durch schwer operationalisierbare Gegenindikatoren wie etwa mangelnde sprachliche Fähigkeiten verdecken, was zu falschen Schlussfolgerungen im Rahmen der Auswertung führen würde. Dieses Problem ist jedem Ansatz gemein, der auf vorhandene Daten aus der Praxis zurückgreift, statt kontrolliert neue Daten zu erzeugen. Umgekehrt berücksichtigt ein solcher ,historisch-archäologischer“ Ansatz echte Entscheidungen durch echte Beurteiler und Beurteilerinnen, was zumindest den sonst häufigen Kritikpunkt der Künstlichkeit kontern kann (Dhami und Belton 2015, für eine Diskussion der Aktenanalyse als Quelle für die Urteils- und Entscheidungsforschung).

Ohne Frage ist die Modellierung vollzuglicher Selektionsprozesse mit großem Aufwand, vielen Herausforderungen und einigen Sackgassen verbunden. Eine größere Zahl dieser Probleme ist allerdings darauf zurückzuführen, dass die Daten in der Regel nicht für diesen Zweck erhoben wurden und die Modellierung vermutlich nicht mitgedacht worden ist. Ein erster wichtiger Schritt zu einem besseren Verständnis vollzuglicher Prozesse kann daher darin bestehen, die Modellierung dieser Prozesse in der Studienplanung und Operationalisierung zu berücksichtigen.

Als zentrales Argument dieses Beitrags sollte im Übrigen nicht etwa die Kritik an der bisherigen Forschung gesehen werden. Ebenfalls ist es nicht das Anliegen, die hier ver- 
wendete Analysestrategie und das hier entwickelte Modell als Allheilmittel oder der Weisheit letzter Schluss zu präsentieren. Das Hauptargument ist vielmehr, dass es a) gute Argumente gibt, die Urteils- und Entscheidungsprozesse im Justizvollzug zu thematisieren und b) die Psychologie eine Reihe von Theorien und Methoden bereithält, sich dieser Frage zu nähern. Wir plädieren daher dafür, dass trotz der dargestellten Schwierigkeiten in Zukunft häufiger und genauer auf die vollzuglichen Urteils- und Entscheidungsprozesse geschaut wird, um die wichtigen Selektionsprozesse besser verstehen und die Ergebnisse der Evaluationsforschung besser deuten zu können.

Interessenkonflikt M. Guéridon und S. Suhling geben an, dass kein Interessenkonflikt besteht.

Open Access Dieser Artikel wird unter der Creative Commons Namensnennung 4.0 International Lizenz veröffentlicht, welche die Nutzung, Vervielfältigung, Bearbeitung, Verbreitung und Wiedergabe in jeglichem Medium und Format erlaubt, sofern Sie den/die ursprünglichen Autor(en) und die Quelle ordnungsgemäß nennen, einen Link zur Creative Commons Lizenz beifügen und angeben, ob Änderungen vorgenommen wurden.

Die in diesem Artikel enthaltenen Bilder und sonstiges Drittmaterial unterliegen ebenfalls der genannten Creative Commons Lizenz, sofern sich aus der Abbildungslegende nichts anderes ergibt. Sofern das betreffende Material nicht unter der genannten Creative Commons Lizenz steht und die betreffende Handlung nicht nach gesetzlichen Vorschriften erlaubt ist, ist für die oben aufgeführten Weiterverwendungen des Materials die Einwilligung des jeweiligen Rechteinhabers einzuholen.

Weitere Details zur Lizenz entnehmen Sie bitte der Lizenzinformation auf http://creativecommons.org/licenses/by/4.0/deed.de.

\section{Literatur}

Beckstead JW (2017) The bifocal lens model and equation: examining the linkage between clinical judgments and decisions. Med Decis Making 37(1):35-45

Bonta J, Andrews DA (2017) The psychology of criminal conduct. Routledge, London

Bröder A (2000) A methodological comment on behavioral decision research. Psychol Beitr 42(4):645-662

Bröder A, Hilbig BE (2017) Urteilen und Entscheiden. In: Müsseler J, Rieger M (Hrsg) Allgemeine Psychologie. Springer, Heidelberg, Berlin, New York

Brunswik E (1955) Representative design and probabilistic theory in a functional psychology. Psychol Rev 62(3):193-217
Calliess R-P, Müller-Dietz H (2005) Strafvollzugsgesetz. C.H.Beck, München

Cooksey RW (1996) Judgment analysis: theory, methods, and applications. Academic, San Diego

Dahle KP (2005) Psychologische Kriminalprognose: Wege zu einer integrativen Methodik für die Beurteilung der Rückfallwahrscheinlichkeit bei Strafgefangenen. Centaurus, Freiburg

Dhami M, Belton I (2015) Using court records for sentencing research: pitfalls and possibilities. In: Roberts JV (Hrsg) Exploring sentencing practice in England and Wales. Palgrave Macmillan, London

Egg R (2013) § 9 Verlegung in eine sozialtherapeutische Anstalt. In: Schwind H-D, Böhm A, Jehle J-M, Laubenthal K (Hrsg) Strafvollzugsgesetz: Bund und Länder. de Gruyter, Berlin

Endres J (2014) Determinanten der Behandlungsteilnahme und des Behandlungsabbruchs bei inhaftierten Sexualstraftätern. Forum Strafvollzug 40(3):237-243

Gigerenzer G (2006) Heuristics. In: Gigerenzer G, Engel C (Hrsg) Heuristics and the law. MIT Press, Cambridge

Goldstein WM (2004) Social judgment theory: applying and extending Brunswik's probabilistic functionalism. In: Koehler DJ, Harvey N (Hrsg) Handbook of judgment and decision making. Blackwell, Malden, S 37-61

Hamm RM, Yang H (2017) Alternative lens model equations for dichotomous judgments about dichotomous criteria. J Behav Decis Making 30(2):527-532

Hammond KR (1996) Human judgment and social policy: irreducible uncertainty, inevitable error, unavoidable injustice. Oxford University Press, New York

Heckman JJ, Smith JA (2004) The determinants of participation in a social program: evidence from a prototypical job training program. J Labor Econ 22(2):243-298

Krohne HW, Hock M (2015) Psychologische Diagnostik: Grundlagen und Anwendungsfelder. Kohlhammer, Stuttgart

Laubenthal K (2015) Strafvollzug. Springer, Berlin

Lösel F, Link E, Schmucker M, Bender D, Breuer M, Carl L, Endres J, Lauchs L (2019) On the effectiveness of sexual offender treatment in prisons: a comparison of two different evaluation designs in routine practice. Sex Abuse. https://doi.org/10.1177/ 1079063219871576

Rettenberger M, von Franqué F (2013) Handbuch kriminalprognostischer Verfahren. Hogrefe, Göttingen

Seifert S (2014) Der Umgang mit Sexualstraftätern: Bearbeitung eines sozialen Problems im Strafvollzug und Reflexion gesellschaftlicher Erwartungen. Springer VS, Wiesbaden

Stewart TR (2001) The lens model equation. In: Hammond KR, Stewart TR (Hrsg) The essential Brunswik: beginnings, explications, applications. Oxford University Press, New York

Suhling S, Wischka B (2008) Indikationskriterien für die Verlegung von Sexualstraftätern in eine sozialtherapeutische Einrichtung. Monatsschr Kriminol Strafrechtsreform 91:210-226 